\title{
Blackbody Fits of Semiregular Variables
}

\author{
F. Kerschbaum and J. Hron \\ Institut für Astronomie der Universität Wien, \\ Türkenschanzstraße 17, A-1180 Wien, Austria, \\ Offprints: kerschbaum@astro.ast.univie.ac.at
}

\begin{abstract}
Semiregular variables of type SRa and SRb are studied by fitting combinations of blackbodies to new near infrared and IRAS data.
\end{abstract}

In Kerschbaum \& Hron (1992) a systematic investigation of the basic properties of type SRa and SRb Semiregular variables (SRVs) was presented. For the Orich subgroup a new classification scheme into 'blue', 'red' and 'Mira' SRVs was one of the main outcomes. Concerning the physical properties an independent study by Jura \& Kleinmann (1992) arrived at similar conclusions, except for the long-period SRVs. New JHKL'M-photometry (Kerschbaum \& Hron 1994, Paper II) supports independently the classification into 'red' and 'blue' SRVs.

As a next step, visual information is taken from the GCVS4, near infrared JHKL'M-photometry from Paper II; mid- and far-infrared measurements were extracted from the IRAS-PSC. The resulting energy distributions can be reasonably well fitted for almost all objects by a combination of two blackbody curves. The overall spectra can be roughly described by a 'photospheric' temperature $T^{*}$, a 'dust'-temperature $T^{d}$ and the 'size' of the circumstellar shell relative to the star $r=R^{d} / R^{*}$.

The resulting parameters $T^{*}, T^{d}$ and $r$ of course need to be related to astrophysically meaningful quantities. $T^{*}$ can be compared with a $T_{\text {eff }}$ derived from the synthetic $\mathrm{H}-\mathrm{K}$ colours given by Bessell et al. (1989). For $T^{*}$ above $2100 \mathrm{~K}$ only a constant offset of about $400 \mathrm{~K}$ is found for our O-rich SRVs. For the parameters related to the circumstellar material, namely $T^{d}$ and $r=R^{d} / R^{*}$ the situation is more complex. A comparison of BB-Fits with the results of dust shell models by Le Bertre (1988) and Le Sidaner \& Le Bertre (1993) shows that our $T^{d}$ and $r$ both correspond to almost the same region of the innermost part of the dust shell.

Fig. 1 shows the blackbody fit parameters for 'red' SRVs and C- and SSRVs. The different energy distributions show up clearly. All 'blue' objects can be reasonably well approximated by only one blackbody whereas the 'red' and the 'Mira' SRVs need two. The $T^{*}$ values, reflecting mainly the effective temperatures for objects with only small mass loss, are typically $300 \mathrm{~K}$ higher in the 'blue' than in the 'red' SRV cases. With a few extreme exceptions most of the 'red' $T^{d}$ values are concentrated around $400 \mathrm{~K}$ whereas the 'Mira' like Semiregular variables show a wider distribution between 330 and $570 \mathrm{~K}$. C-rich objects and S-stars differ significantly from the O-SRVs in the fit parameters. Sometimes 'unphysically' low $T^{*}$ are found - a result of circumstellar reddening in the high mass loss cases. Also lower values of $T^{d}$, accompanied by :ormal $T^{*}$ 

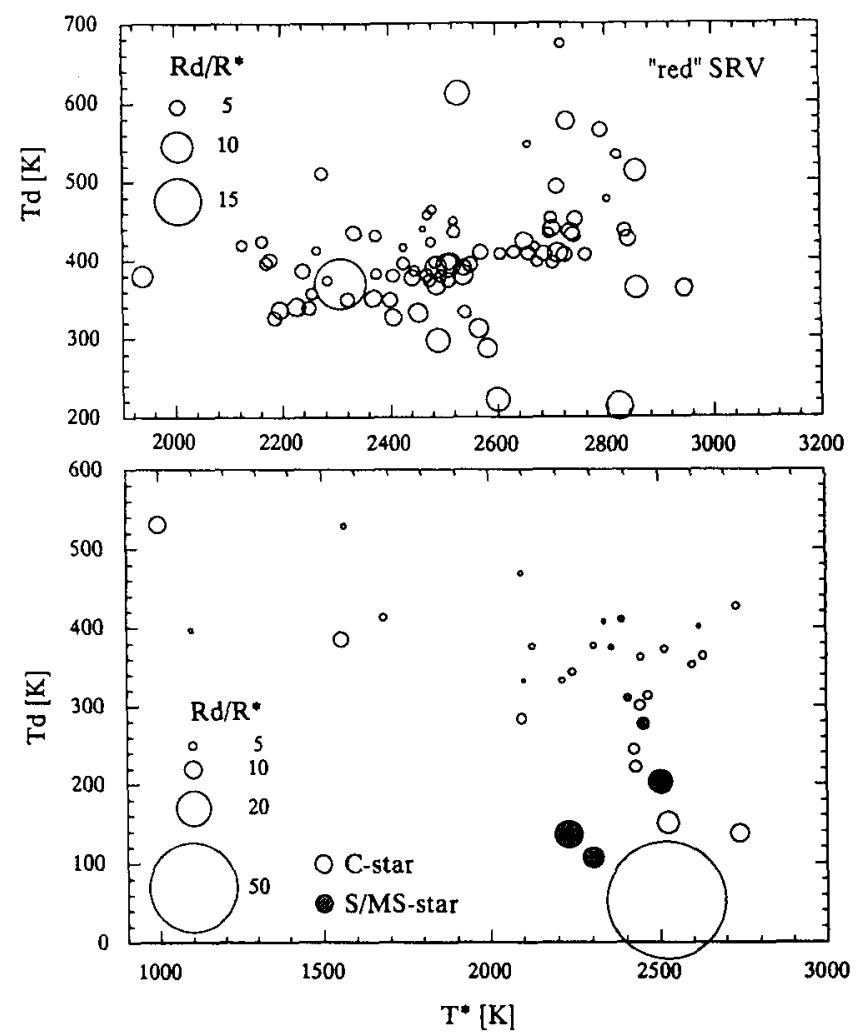

Figure 1. 'Dust'-temperature $T^{d}$ as a function of 'photospheric' temperature $T^{*}$ with the relative 'size' of the dust shell $R^{d} / R^{*}$ indicated by the diameter of the plot symbols.

and large values of $r$ are common - the most extreme example being TT Cyg with its detached envelope.

Acknowledgments. This work was supported by the ASF under project numbers P7252-PHY and P9638-AST.

\section{References}

Bessell M.S., Brett J.M., Scholz M., Wood P.R. 1989, A\&AS 77, 1

Jura M. 1988, ApJS 66, 33

Jura M., Kleinmann S.G. 1992, ApJS 79, 105

Kerschbaum F., Hron J. 1992, A\&A 263, 97 (Paper I)

Kerschbaum F., Hron J. 1994, A\&AS 106, 397 (Paper II)

Le Bertre T. 1988, A\&A 190, 79

Le Sidaner P., Le Bertre T. 1993, A\&A 278, 167 